\title{
Prevalência de anemia e sua associação com aspectos sociodemográficos e antropométricos em crianças de Vitória, Espírito Santo, Brasil
}

\author{
The prevalence of anemia and its association \\ with socio-demographic and anthropometric aspects \\ in children living in Vitória, State of Espírito Santo, Brazil
}

Ana Paula Della Nina de Oliveira ${ }^{1}$

Mariana Nunes Pascoal ${ }^{1}$

Luana Caroline dos Santos ${ }^{1}$

Simone Cardoso Lisboa Pereira ${ }^{1}$

Liusani Elizia Honório Justino ${ }^{2}$

Glenda Blaser Petarli ${ }^{3}$

Pedro Makumbundu Kitoko ${ }^{2}$

\footnotetext{
${ }^{1}$ Escola de Enfermagem, Universidade Federal de Minas Gerais. Av. Alfredo Balena 190, Centro. 30.130-100 Belo Horizonte MG Brasil.

anadellanina@gmail.com

${ }^{2}$ Conselho de Segurança

Alimentar e Nutricional do

Espírito Santo.

${ }^{3}$ Hospital Universitário

Cassiano Antônio de

Morais.
}

\begin{abstract}
The high prevalence of iron deficiency in the population is widely acknowledged, but there are not yet sufficient studies that explain the reasons for this problem at local and national level. Thus, the scope of this study was to evaluate the prevalence of anemia and its relation with sociodemographic and anthropometric aspects of children living in Vitoria, Espirito Santo. This is a cross-sectional study conducted among 12- to 72month-old children. Data were collected in Primary Healthcare Units and involved the hemoglobin dosage, height and weight measurement, in addition to the application of a semi-structured questionnaire to gather socio-demographic information. The evaluation included 980 children, $51.1 \%$ of which were female with a median age of 46.00 (95\% CI: 45.52 - 48.09) months. Anemia was found in 37\%, with a hemoglobin median of 11.4 (95\%CI: 11.15 - 11.36) g/dL. The children's' height/ age ratio $(p=0.049)$ and the number of people in the family $(p<0.001)$ were found to be determinants of anemia, and age was a factor of protection for this deficiency $(p=0.010)$. The high prevalence of anemia and its strong relation with demographic and anthropometric data suggest the need for more effective public policies than just food fortification and supplementation.
\end{abstract}

Key words Anemia, Child health, Socioeconomic factors, Child well-being, Population characteristics
Resumo É reconhecida a alta prevalência da deficiência de ferro na população, mas ainda não existem estudos suficientes que expliquem os motivos deste problema em nivel local e nacional. Assim, o objetivo deste estudo foi avaliar a prevalência de anemia e sua relação com aspectos sociodemográficos e antropométricos de crianças residentes no município de Vitória, ES. Trata-se de um estudo transversal realizado com indivíduos de 12 a 72 meses, cujos dados foram coletados em Unidades Básicas de Saúde e envolveu a dosagem de hemoglobina, aferição de peso e estatura, além de aplicação, aos responsáveis legais, de questionário semiestruturado com informações sociodemográficas. Foram avaliadas 980 crianças, $51,1 \%$ do sexo feminino e mediana de idade de 46,00 (IC95\%: 45,52-48,09) meses. Constatou-se 37\% de anemia, sendo 11,4 (IC95\%: 11,15-11,36) g/dL o valor mediano de hemoglobina. A estatura/idade da crian$c ̧ a(p=0,049)$ e o número de pessoas na família ( $p$ $<0,001)$ apresentaram-se como determinantes da anemia, e a idade como fator protetor desta carência $(p=0,010)$. A elevada prevalência de anemia e sua forte relação com dados sociodemográficos e antropométricos sugerem a necessidade de politicas públicas mais efetivas do que apenas a fortificação e a suplementação de alimentos.

Palavras-chave Anemia, Saúde da criança, Fatores socioeconômicos, Bem-estar da criança, $\mathrm{Ca}$ racterísticas da população 


\section{Introdução}

Apesar das mudanças epidemiológicas observadas nas últimas décadas, a deficiência de ferro ainda constitui-se como um problema de saúde pública que afeta países desenvolvidos e em desenvolvimento e que tem como principais grupos de risco lactentes, pré-escolares, mulheres em idade fértil e gestantes ${ }^{1,2}$. A anemia (baixa concentração de hemoglobina no sangue), decorrente de reservas insuficientes de ferro, pode resultar em prejuízos funcionais ao organismo, como retardo do desenvolvimento infantil, comprometimento da imunidade celular e diminuição da capacidade intelectual ${ }^{3,4}$. Na infância, três fatores contribuem para a alta prevalência de anemia: reservas insuficientes de ferro ao nascer, principalmente em prematuros e nascidos com baixo peso; elevada velocidade de crescimento; e balanço negativo entre a ingestão e perdas do mineral ${ }^{5}$.

Segundo um levantamento realizado pela Organização Mundial da Saúde (OMS) entre 1993 e 2005 em 193 países de diferentes níveis de desenvolvimento, em $78 \%$ destes locais a prevalência de anemia em crianças com idade inferior a 5 anos corresponde a, no mínimo, $20 \%{ }^{6}$. Adicionalmente, foi identificada prevalência de $47,4 \%$ de anemia entre pré-escolares em âmbito mundial, e de $54,9 \%$ no Brasil, sendo esta a segunda maior da América Latina ${ }^{7}$. Esses valores são de grande relevância tendo em vista que a OMS considera como situação de saúde pública severa as prevalências de anemia iguais ou superiores a $40 \%$, moderadas de 20 a $39,9 \%$, leves de 5 a $19,9 \%$, e normais aquelas inferiores a $5 \%{ }^{2}$.

No município de Vitória, Espírito Santo, os estudos são escassos, mas denotam a importância do problema. Almeida et al. ${ }^{8}$ avaliaram 760 crianças de Centros Municipais de Educação Infantil, com idade de 6 meses a 7 anos, e observaram que $25 \%$ apresentavam anemia. Porém, não existem estudos suficientes que expliquem os motivos da alta prevalência do problema em nível local e nacional, dificultando a elaboração de ações de intervenção e controle da situação9.

Alguns estudos têm ainda relacionado a prevalência de anemia com o estado nutricional e aspectos sociodemográficos em crianças ${ }^{10-15}$, sendo tais fatores possíveis determinantes de tal carência nesta população. Isto, somado às consequências da anemia na infância e ao baixo número de estudos sobre assunto no Brasil, denota a importância de investigações mais abrangentes na área. Dessa maneira, o presente estudo teve como objetivo avaliar a prevalência de anemia e sua relação com aspectos sociodemográficos e antropométricos de crianças residentes no município de Vitória, ES.

\section{Métodos}

Trata-se de um estudo transversal realizado com crianças de 12 a 72 meses de idade, residentes no município de Vitória, Espírito Santo. Os dados foram coletados entre os meses de Abril e Agosto de 2008, com auxílio de equipe devidamente treinada. A mobilização foi realizada por Agentes Comunitários de Saúde (ACS), os quais convidavam os pais ou responsáveis legais a comparecerem com as crianças à Unidade Básica de Saúde (UBS) de sua área.

O município de Vitória é dividido em oito regiões administrativas - Jardim Camburí, Maruípe, São Pedro, Santo Antônio, Jucutuquara/ Bento Ferreira, Continental, Praia do Canto e Centro - que, por sua vez, são divididas em vinte e oito áreas de saúde, cada uma representada por uma UBS. Dentre as regiões, São Pedro, Santo Antônio e Maruípe apresentam menores indicadores socioeconômicos, como o Índice de Qualidade Urbana $(0.22-0.44 ; 0.42-0.55 ; 0.20-0.71$, respectivamente) ${ }^{16}$.

A população total de crianças entre 12 e 72 meses residente em toda a capital é estimada em $26.967^{18}$. O desenho amostral foi realizado considerando-se uma margem de erro de $5 \%$ e poder do teste de $95 \%$, o que significou um mínimo de 395 crianças, distribuídas de acordo com a proporção da população nessa faixa etária em cada região administrativa (Tabela 1$)^{17}$.

A coleta de dados realizou-se nas UBS de cada área de saúde incluída no estudo e consistiu de aplicação de questionário semiestruturado com informações sociodemográficas e antropométricas. Verificou-se a idade e o sexo da criança, bem como a idade e a escolaridade materna, o número de pessoas na família e os níveis socioeconômicos (A1, A2, B1, B2, C, D, E), classificados segundo o grau de instrução do chefe da família e o número de itens de bens de consumo existentes na casa dos entrevistados ${ }^{18}$.

Além disso, avaliou-se peso e estatura de acordo com as recomendações do Ministério da Saú$\mathrm{de}^{19}$, sendo as medidas antropométricas aferidas em duplicata, com posterior cálculo da média das mensurações obtidas. A classificação da antropometria foi realizada segundo recomendações do Ministério da Saúde ${ }^{19}$ com auxílio das curvas de crescimento dos índices altura/idade e 
Tabela 1. Caracterização da amostra estudada segundo região administrativa de saúde. Vitória-ES, Brasil, 2008.

\begin{tabular}{lccccc}
\hline \multirow{2}{*}{$\begin{array}{c}\text { Região administrativa } \\
\text { de saúde }\end{array}$} & \multicolumn{2}{c}{ Amostra de crianças calculada } & & Amostra de crianças avaliadas \\
\cline { 6 - 6 } \cline { 5 - 6 } & $\mathbf{N}$ & $\%$ & & N & $\%$ \\
\hline Maruípe & 68 & 17,2 & & 204 & 20,8 \\
Continental & 66 & 16,6 & & 104 & 10,6 \\
São Pedro & 66 & 16,6 & & 164 & 16,7 \\
Santo Antônio & 45 & 11,7 & 181 & 18,5 \\
Bento Ferreira/Jucutuquara & 42 & 10,6 & & 138 & 14,1 \\
Praia do Canto & 42 & 10,6 & & 36 & 03,7 \\
Jardim Camburí & 33 & 08,3 & & 46 & 05,1 \\
Centro & 33 & 08,3 & & 107 & 10,9 \\
Total & 395 & 100,0 & & 980 & 100,0 \\
\hline
\end{tabular}

Índice de Massa Corporal (IMC)/idade, utilizando-se pontos de corte em escore $\mathrm{z}^{19-21}$.

Para a obtenção dos dados de anemia, foram coletados de cada criança $30 \mu \mathrm{L}$ de sangue, com auxílio de microlancetas descartáveis, e depositados diretamente em uma microcuveta, com posterior leitura por aparelho de fotometria portátil Hemocontrol $^{\circledast}$ para dosagem da hemoglobina. Utilizou-se, como ponto de corte, o valor de 11,0 $\mathrm{g} / \mathrm{dL}$ para a definição de anemia em criança de 12 a 59 meses e 11,5 g/dL para aquelas de 60 a 72 meses de idade ${ }^{5}$, além da classificação proposta por DeMayer et al. ${ }^{22}$ para a determinação do grau da anemia como leve (de 9,0 a 10,9/11,4 g/dL), moderada (de 7,0 a $8,9 \mathrm{~g} / \mathrm{dL}$ ) ou severa $(<7,0 \mathrm{~g} / \mathrm{dL})$.

Os dados obtidos foram digitados no Programa $\mathrm{Excel}^{\circledR} 2003$ e posteriormente analisados com auxílio do Software Statistical Package for the Social Sciences (SPSS) versão 17.0. Foi realizada análise descritiva dos dados, com cálculo das distribuições de frequências, medidas de tendência central e de dispersão. Adicionalmente, foram realizados os testes de normalidade Kolmogorov-Smirnov, bem como Correlação de Pearson e Spearman para avaliar a relação entre variáveis contínuas, t de Student Simples e análise de variância (ANOVA) - com posterior teste de Tukey para a comparação entre subgrupos para comparação de médias, e Mann-Whitney e Kruskal-Wallis para comparação de medianas.

Realizou-se ainda teste exato de Fisher para avaliar associações entre as variáveis, e análise de regressão logística para verificar associações entre fatores determinantes com a ocorrência e grau de anemia, utilizando-se critério Stepwise de seleção de variáveis.

Os resultados foram apresentados na forma de medianas e intervalos de confiança (IC95\%) para as variáveis quantitativas, além de $O d d s R a$ tio e intervalos de confiança (IC95\%) para variáveis determinantes da ocorrência e grau de anemia. Adotou-se valor de significância de 5\%.

Respeitando a integridade e a dignidade dos sujeitos do estudo, todos os responsáveis legais das crianças receberam e assinaram um termo de consentimento livre e esclarecido, sendo as anêmicas encaminhadas ao atendimento especializado na UBS mais próxima à sua residência. Esta pesquisa foi aprovada pelo Comitê de Ética em Pesquisa da Faculdade Salesiana de Vitória e contou com o apoio da Fundação de Apoio à Ciência e Tecnologia do Espírito Santo (FAPES).

\section{Resultados}

Foram avaliadas 980 crianças, $51,1 \%$ do sexo feminino, contemplando $3,6 \%$ da população entre 12 a 72 meses do município de Vitória. As regiões foram adequadamente representadas de acordo com sua população infantil, porém a amostra mostrou-se menor que o esperado nas regiões de Jardim Camburí e Continental e não foi representativa na Praia do Canto, onde ocorreram dificuldades de adesão à coleta de dados (Tabela 1).

A mediana de idade das crianças estudadas foi de 46,00 (IC95\%: 45,52 - 48,09) meses, e observou-se que a maioria pertencia aos níveis socioeconômicos C e D (46,6\% e 42,9\%, respectivamente). A mediana de idade materna foi de 28,00 (IC95\%: 28,15 - 29,05) anos, sendo que 35\% possuíam apenas o ensino fundamental completo, $16,4 \%$ o ensino médio completo e somente $1,7 \%$ das mães completaram o ensino superior. O número mediano de pessoas na família foi de 4,00 (IC95\%: 4,71 - 4,94), Tabela 2. 
Tabela 2. Características sociodemográficas e antropométricas de crianças de 12 a 72 meses. Vitória (ES), Brasil, 2008.

\begin{tabular}{|c|c|c|}
\hline \multicolumn{3}{|c|}{ Características } \\
\hline Variáveis & Mediana & Intervalo de Confiança (IC95\%) \\
\hline Idade (meses) & 46,00 & $45,52-48,09$ \\
\hline Idade materna (anos) & 28,00 & $28,15-29,05$ \\
\hline Pessoas na família & 4,00 & $4,71-4,94$ \\
\hline Sexo & \multicolumn{2}{|r|}{ (5 } \\
\hline Feminino & \multicolumn{2}{|r|}{51,1} \\
\hline Masculino & \multicolumn{2}{|r|}{48,9} \\
\hline Classe Social & \multicolumn{2}{|r|}{$\%$} \\
\hline A & \multicolumn{2}{|r|}{1,3} \\
\hline B & \multicolumn{2}{|r|}{7,4} \\
\hline $\mathrm{C}$ & \multicolumn{2}{|r|}{46,6} \\
\hline $\mathrm{D}$ & \multicolumn{2}{|r|}{41,9} \\
\hline E & \multicolumn{2}{|r|}{2,8} \\
\hline Escolaridade materna & \multicolumn{2}{|r|}{$\%$} \\
\hline Fundamental incompleto & \multicolumn{2}{|r|}{2,1} \\
\hline Fundamental completo & \multicolumn{2}{|r|}{35,0} \\
\hline Médio incompleto & \multicolumn{2}{|r|}{13,1} \\
\hline Médio completo & \multicolumn{2}{|r|}{16,4} \\
\hline Superior incompleto & \multicolumn{2}{|r|}{29,0} \\
\hline Superior completo & \multicolumn{2}{|r|}{1,7} \\
\hline Técnico & \multicolumn{2}{|r|}{2,7} \\
\hline Classificações antropométricas & \multicolumn{2}{|r|}{$\%$} \\
\hline \multicolumn{3}{|l|}{ Estatura para a idade } \\
\hline Estatura adequada para a idade & \multicolumn{2}{|r|}{92,9} \\
\hline Baixa estatura para a idade & \multicolumn{2}{|r|}{7,1} \\
\hline \multicolumn{3}{|l|}{ Índice de Massa Corporal para a idade } \\
\hline Baixo Índice de Massa Corporal para idade & \multicolumn{2}{|r|}{3,5} \\
\hline Eutrofia & \multicolumn{2}{|r|}{73,9} \\
\hline Sobrepeso & \multicolumn{2}{|r|}{15,8} \\
\hline Obesidade & \multicolumn{2}{|r|}{6,8} \\
\hline
\end{tabular}

Em relação à avaliação antropométrica, observou-se que $7,1 \%$ das crianças apresentaram baixa estatura para a idade, $22,6 \%$ estavam com excesso de peso (15,8\% sobrepeso e $6,8 \%$ obesidade) e 3,5\% possuíam IMC baixo para a idade (Tabela 2).

Quanto aos dados bioquímicos, constatouse $37 \%$ de anemia na população estudada, cujo valor mediano de hemoglobina foi de $11,4 \mathrm{~g} / \mathrm{dL}$ (IC95\%: 11,15 - 11,36 g/dL). Dos 363 casos observados, $18,2 \%$ apresentava-se em sua forma moderada e $1,9 \%$ na forma severa. O grau de anemia associou-se à faixa etária, estatura por idade e nível socioeconômico, sendo os maiores percentuais de anemia leve, moderada e severa pertencentes às crianças de menor faixa etária ( $\mathrm{p}$ $<0,001)$, com baixa estatura para idade ( $\mathrm{p}=$ $0,014 ; 0,017)$ e pertencentes aos níveis C, D e E ( $\mathrm{p}$ $<0,001 ; 0,003 ; 0,011)$, Tabela 3.
Os valores de hemoglobina apresentaram correlação com a idade das crianças $(r=0,256$; $p$ $<0,001)$, número de pessoas na família $(\mathrm{r}=$ $0,68 ; \mathrm{p}=0,035)$ e IMC $(\mathrm{r}=-0,101 ; \mathrm{p}=0,002)$. Ademais, diferiu segundo as regiões administrativas de saúde ( $\mathrm{p}=0,003)$, com menores valores em Maruípe (11,30; IC95\%: 10,84 - 11,26 g/dL) e valores mais elevados em Jardim Camburí (11,95; IC95\%: 11,37 - 12,16 g/dL; p = 0,001). Nas demais regiões não houve diferença estatisticamente significante.

A estatura/idade da criança e o número de pessoas na família apresentaram-se como determinantes da anemia e a idade como fator protetor desta carência. Os indivíduos com baixa estatura apresentaram 76 vezes o risco de serem anêmicos quando comparados aos de estatura adequada ( $\mathrm{p}=0,049)$, e o risco desta carência nutricional e na sua forma severa aumentou 
$11,5 \%(p<0,001)$ e $9,4 \%(p=0,020)$, respectivamente, ao acréscimo de 1 pessoa na família ( $\mathrm{p}<$ $0,001)$ e reduziu $4,2 \%(\mathrm{p}=0,010)$ e $3,9 \%(\mathrm{p}<$ $0,001)$, respectivamente, a cada acréscimo de um mês de idade, Tabela 4.

\section{Discussão}

O estudo foi conduzido com um número de crianças muito maior em relação ao estipulado pelo cálculo amostral, em virtude da forma de recrutamento por livre demanda, buscando-se maior representatividade de cada região administrativa do município de Vitória. Porém, essa representatividade não foi alcançada na Praia do Canto e o número de avaliados foi menor que o esperado em Jardim Camburí e Continental, o que pode ser atribuído às melhores condições sociodemográficas destas regiões, reduzindo a frequência às Unidades Básicas de Saúde, pouco utilizadas por pessoas de nível social mais elevado ${ }^{16,23}$.

Observou-se que a maioria das famílias analisadas pertencia às classes $\mathrm{C}$ e $\mathrm{D}$, além de apresentarem baixo nível de escolaridade materna, o que pode estar relacionado às características da população comumente frequentadora de serviço público de saúde, local utilizado para a coleta de dados $^{23}$.

Quanto à antropometria, a prevalência de crianças com déficit de estatura para a idade foi semelhante aos dados nacionais $(7 \%)^{24}$, e maior que

Tabela 3. Fatores associados ao grau de anemia de crianças de 12 a 72 meses. Vitória (ES), Brasil, 2008.

\begin{tabular}{|c|c|c|c|c|c|}
\hline Variáveis & $\begin{array}{c}\text { Sem anemia } \\
(\%)\end{array}$ & $\begin{array}{l}\text { Anemia } \\
\text { leve }(\%)\end{array}$ & $\begin{array}{c}\text { Anemia moderada } \\
(\%)\end{array}$ & $\begin{array}{c}\text { Anemia severa } \\
(\%)\end{array}$ & Valor $\mathrm{p}$ \\
\hline \multicolumn{6}{|l|}{ Idade (meses) } \\
\hline $12-24$ & $37,8^{\text {abcdef }}$ & $46,6^{\text {ace }}$ & $13,5^{\mathrm{bdf}}$ & 2,1 & $<0,001^{\mathrm{ab}}$ \\
\hline $25-36$ & $50,9^{\mathrm{gh}}$ & $39,5^{\mathrm{gh}}$ & 5,9 & 3,7 & $<0,001^{\mathrm{cd}}$ \\
\hline $37-48$ & $70,8^{\mathrm{ab}}$ & $23,3^{\mathrm{a}}$ & $5,4^{\mathrm{b}}$ & 0,5 & $<0,001^{\text {ef }}$ \\
\hline $49-60$ & $71,4^{\mathrm{cdg}}$ & $22,0^{\mathrm{cg}}$ & $6,0^{\mathrm{d}}$ & 0,6 & $<0,001^{\mathrm{g}}$ \\
\hline $61-72$ & $65,9^{\text {efh }}$ & $27,1^{\text {eh }}$ & $4,1^{\mathrm{f}}$ & 2,9 &, $005^{\mathrm{h}}$ \\
\hline \multicolumn{6}{|c|}{ Estatura por idade } \\
\hline Adequada & $63,1^{\mathrm{a}}$ & 29,3 & $7,0^{\mathrm{b}}$ & $0,6^{\mathrm{ab}}$ &, $014^{\mathrm{a}}$ \\
\hline Baixa & $52,2^{\mathrm{a}}$ & 40,6 & $4,3^{\mathrm{b}}$ & $2,9^{\mathrm{ab}}$ &, $017^{\mathrm{b}}$ \\
\hline \multicolumn{6}{|l|}{ Classe social } \\
\hline A 1 & $80,6^{\mathrm{abcde}}$ & $19,4^{\mathrm{abcde}}$ & $0,0^{\text {bcde }}$ & $0,0^{\mathrm{e}}$ & $<0,001^{\text {acd }}$ \\
\hline A 2 & $66,7^{\text {afg }}$ & $33,3^{\text {afg }}$ & $0,0^{\mathrm{fg}}$ & $0,0^{\mathrm{g}}$ &, $005^{\mathrm{fg}}$ \\
\hline B1 & $75,0^{\text {hi }}$ & $25,0^{\mathrm{hi}}$ & $0,0^{\mathrm{hi}}$ & $0,0^{\mathrm{i}}$ &, $003^{\mathrm{hi}}$ \\
\hline B2 & $64,4^{\mathrm{bj}}$ & $30,5^{\mathrm{bj}}$ & $5,1^{\mathrm{b}}$ & 0,0 & $<0,001^{\mathrm{j}}$ \\
\hline $\mathrm{C}$ & $66,9^{\mathrm{ckl}}$ & $26,6^{\mathrm{cl}}$ & $6,3^{\mathrm{c}}$ & $0,2^{\mathrm{kl}}$ &, $001^{1}$ \\
\hline $\mathrm{D}$ & $57,4^{\mathrm{dfhk}}$ & $33,5^{\mathrm{dfh}}$ & $8,1^{\text {adfh }}$ & $1,0^{\mathrm{k}}$ &, $003^{\mathrm{k}}$ \\
\hline $\mathrm{E}$ & $50,0^{\text {egijl }}$ & $38,5^{\text {egijl }}$ & $7,7^{\text {egi }}$ & $3,8^{\text {egil }}$ &, $011^{\mathrm{e}}$ \\
\hline
\end{tabular}

${ }^{*}$ Letras iguais correspondem a valores que se associam.

Tabela 4. Fatores determinantes de anemia de crianças de 12 a 72 meses. Vitória (ES), Brasil, 2008.

\begin{tabular}{lccc}
\hline \multicolumn{1}{c}{ Variáveis } & Categorias & Odds Ratio & IC95\% \\
\hline Desfecho: Presença de anemia & - & & \\
$\quad$ Idade da criança (anos) & - & 0,958 & $0,946-0,971$ \\
Número de pessoas na família & Estatura adequada para a idade & 1,114 & $1,026-1,210$ \\
Estatura por idade & Baixa estatura para a idade & 1,76 & - \\
& - & 0,961 & $0,949-0,973$ \\
Desfecho: Anemia severa & - & 1,094 & $1,014-1,181$ \\
Idade da criança (anos) & - & & \\
Número de pessoas na família & &
\end{tabular}

* Regressão Logística Multivariada; IC: Intervalo de Confiança 
a verificada em crianças da mesma faixa etária de Pelotas (5\%) em estudo de base populacional de Assunção et al. ${ }^{3}$. Esta diferença pode ser atribuída ao maior número de indivíduos anêmicos diagnosticados no presente estudo (37\% versus 30,2\%), já que a anemia e baixa estatura para a idade estão relacionadas a problemas nutricionais semelhantes, como o consumo insuficiente de nutrientes e a manifestação de doenças infecciosas ${ }^{25}$.

Adicionalmente, o excesso de peso observado foi superior aos achados na literatura $(12 \%)^{3}$ e pela Pesquisa Nacional de Demografia e Saúde da Criança e da Mulher $(7,3 \%)^{26}$, apesar desta característica ser constantemente associada às crianças de classes econômicas mais favorecidas ${ }^{27}$ 29. Contudo, atualmente, elevadas prevalências de sobrepeso e obesidade também têm sido identificadas em classes econômicas e níveis de escolaridade mais baixos, como no presente estudo ${ }^{29}$.

Com relação à anemia, observou-se uma prevalência elevada, demonstrando tratar-se de um problema iminente, apesar da transição nutricional que vem sendo observada na população mundial ${ }^{30}$. De acordo com a classificação da OMS (2001), a situação da anemia em Vitória é considerada um problema moderado de saúde pública, mostrando-se como uma preocupação importante a ser considerada na elaboração de programas e projetos específicos ${ }^{5}$. A prevalência da carência constatada foi maior que a encontrada em crianças brasileiras $(30 \%)^{7}$ e em estudo com indivíduos da mesma faixa etária, também realizado em Vitória, no ano de 2004, o que pode significar o aumento do número de crianças anêmicas no município, desde então ${ }^{8}$. Estudos conduzidos em Pernambuco, Alagoas, e São Paulo demonstraram prevalência ainda mais elevada de anemia (33\%; 45,0\% e 68,8\%; respectivamente) $)^{12,31,32}$.

Observou-se que os níveis de hemoglobina foram estatisticamente menores em crianças mais novas, corroborando diversos estudos com indivíduos da mesma faixa etária ${ }^{3,4,8,12,31}$. Ademais, a idade apresentou-se como um fator protetor da ocorrência de anemia, o que pode ser explicado pela elevada variedade alimentar de crianças mais velhas, possuindo maiores possibilidades de consumo de vitaminas e minerais.

A concentração de hemoglobina apresentou relação significativa com fatores sociodemográficos, sendo menor em crianças pertencentes às famílias maiores e ao nível socioeconômico C, D e E. Vieira et al. ${ }^{31}$, em investigação de 666 crianças alagoanas de 6 a 60 meses, constataram maior prevalência de anemia naquelas pertencentes a famílias com 5 ou mais moradores $(49,2 \%)$ e com menor renda per capita $(53,3 \%)$, confirmando dados do presente estudo.

Analisando-se investigações na cidade de São Paulo (1984 - 1996), Monteiro et al. ${ }^{33}$ observaram a tendência de aumento da anemia, mais intensamente em menores estratos econômicos da população, uma vez que melhores condições financeiras relacionam-se a maior acesso a alimentos, e o menor número de filhos está ligado a melhores cuidados com a saúde e a alimentação da criança. Isto explica as diferenças entre as medianas de hemoglobina encontradas nas regiões administrativas de Vitória (ES), sendo observadas menores concentrações nas regiões de menores níveis sociodemográficos ${ }^{16}$.

Constatou-se ainda, no presente estudo, que crianças com maiores índices de massa corporal apresentaram menores concentrações de hemoglobina, o que pode estar relacionado ao consumo alimentar inadequado desses indivíduos, com ingestão excessiva de alimentos ricos em açúcares e gorduras e insuficiente daqueles fontes de vitaminas e minerais ${ }^{34}$. Outra possível explicação seria que crianças obesas poderiam apresentar um estado inflamatório com elevada liberação de citocinas, as quais reduziriam a utilização do ferro pelo organismo, causando hipoferremia. Neste caso, a Interleucina-6 (IL-6) teria um importante papel no estímulo da produção da hepcidina, hormônio responsável pela redução da liberação de ferro pelos enterócitos e macrófagos e, consequentemente, pela diminuição da absorção do mineral pelo organismo ${ }^{35-37}$.

Em contrapartida, observou-se maior prevalência de anemia, principalmente na sua forma severa, em crianças com baixa estatura para a idade, corroborando dados de Rocha et al. ${ }^{38}$. Tal associação pode estar relacionada ao fato de que tanto a anemia quanto o déficit estatural possuem fatores de risco ligados à baixa condição socioeconômica $^{4,10,12,15,38}$.

Este estudo apresenta como limitação a avaliação de apenas um parâmetro para o diagnóstico de anemia, dificultando a classificação quanto à sua principal causa e detecção de possíveis relações com outros aspectos. Apesar disso, foi possível avaliar a prevalência de anemia de amostra representativa de crianças do município de Vitória, bem como verificar a forte influência de aspectos nutricionais e sociodemográficos na ocorrência da doença. 


\section{Conclusões}

O presente estudo permitiu constatar elevada prevalência de anemia ainda presente no município estudado, apesar da transição nutricional vigente, relacionando-se com dados sociodemográficos e antropométricos. Sugere-se, assim, que programas sociais, com educação ambiental e nutricio- nal, possam ser opções de complemento aos programas já existentes, reduzindo o número de casos e melhorando a saúde da população infantil.

Adicionalmente, por meio dos resultados obtidos, torna-se possível alertar sobre a necessidade de desenvolvimento de políticas públicas mais efetivas, que contemplem mais do que programas de fortificação e suplementação alimentar.

\section{Colaboradores}

MN Pascoal trabalhou na análise e interpretação dos dados, bem como na redação do artigo; APDN Oliveira trabalhou da redação e revisão crítica do artigo; SCL Pereira trabalhou na concepção e delineamento, além da revisão crítica do artigo; LC Santos auxiliou na análise dos dados e redação do artigo; LEH Justino, GB Petarli e PM Kitoko participaram da concepção e delineamento do artigo.

\section{Referências}

1. Carvalho MC, Baracat ECE, Sgarbieri VC. Anemia Ferropriva e Anemia de Doença Crônica: Distúrbios no Metabolismo de Ferro. Segurança Alimentar e Nutricional 2006; 13(2):54-63.

2. World Health Organization (WHO). Vitamin and mineral nutrition information system. WHO global database on Vitamin A deficiency. Geneva: WHO; 2006.

3. Assunção MCF, Santos IF, Barros AJD, Gigante DP, Victora CG. Anemia em menores de seis anos: estudo de base populacional em Pelotas, RS. Rev Saude Publica 2007; 41(3):328-335.

4. Santos I, César JA, Minten G, Valle N, Neumann NA, Cercato E. Prevalência e fatores associados à ocorrência de anemia entre menores de seis anos de idade em Pelotas, RS. Rev Bras Epidemiol 2004; 7(4):403-415.

5. World Health Organization (WHO). Iron Deficiency Anaemia: assessment, prevention and control. A guide for programme managers. Geneva: WHO; 2001.

6. World Health Organization (WHO). Centers for Disease Control and Preventions Atlanta. Worldwide prevalence of anaemia 1993-2005. WHO global database on anaemia. Geneva: WHO; 2008.

7. Pan American Health Organization (PAHO). Flour fortification with iron, folic acid and vitamin B12. Santiago: PAHO; 2004.

8. Almeida APC, Zandonade E, Abrantes MM, Lamounier JA. Deficiência de ferro e anemia de crianças de Vitória, ES. J Pediatria 2004; 26(3):140-145.

9. Filho MB, Souza AI, Bresani CC. Anemia como problema de saúde pública: uma realidade atual. Cien Saude Colet 2008; 13(6):1917-1922.

10. Silva LSM, Giugliane ERJ, Aerts DRGC. Prevalência e determinantes de anemia em crianças de Porto Alegre, RS, Brasil. Rev Saude Publica 2001; 35(1):66-73.

11. Silva DG, Franceschini SCC, Priore SE, Ribeiro SMR, Szarfarc SC, Souza SB, Almeida LP, Lima NMM, Maffia UCC. Anemia Ferropriva em crianças de 6 a 12 meses atendidas na rede pública de saúde do município de Viçosa, Minas Gerais. Rev Nutr 2002; 15(3):301-308. 
12. Leal LP, Batista Filho M, de Lira PI, Figueiroa JN, Osório MM. Temporal trends and anaemiaassociated factors in 6- to 59-month-old children in Northeast Brazil. Public Health Nutr 2012; 15(9): 1645-1652.

13. Rocha DM, Lamounier JA, Capanema FD, Franceschini SCC, Norton RC, Costa ABP, Rodrigues MTG, Carvalho MR, Chaves TS. Estado nutricional e prevalência de anemia em crianças que frequentam creches em Belo Horizonte, Minas Gerais. Rev Paul Pediatr 2008; 26(1):6-13.

14. Mondini L, Rodrigues DA, Gimeno SGA, Baruzzi RG. Estado nutricional e níveis de hemoglobina em crianças Aruak e Karibe - povos indígenas do Alto Xingú, Brasil Central, 2001-2002. Rev Bras Epidemiol 2009; 12(3):469-477.

15. Borges CQ, Silva RCR, Assis AMO, Pinto EJ, Fiaccone RL, Pinheiro SMC. Fatores Associados à anemia em crianças e adolescentes de escolas públicas de Salvador, Bahia, Brasil. Cad Saude Publica 2009; 25(4):877-888.

16. Vitória. Prefeitura de Vitória. Secretaria de Gestão Estratégica. Gerência de Informações Municipais. Vitória em Dados, 2006. [acessado 2010 out 25]. Disponível em: www.vitoria.es.gov.br

17. Vitória. Prefeitura de Vitória. Secretaria Municipal de Saúde. A Nova Divisão Territorial. Gerência de Atenção à Saúde, 2006b. [acessado 2010 out 25]. Disponível em: www.vitoria.es.gov.br

18. Instituto Brasileiro de Geografia e estatística (IBGE). Censo demográfico 2000: questionário da amostra. Brasília, 2001. [acessado 2011 set 01]. Disponível em: www.ibge.gov.br

19. Brasil. Ministério da Saúde (MS). Sistema de Vigilância Alimentar e Nutricional (SISVAN). Série A. Normas e Manuais Técnicos. Orientações básicas para coleta, o processamento, a análise de dados e a informação em serviços de saúde. Brasília: MS; 2004.

20. World Health Organization (WHO). Child Growth Standards: length/height-for-age, weight-for-age, weight-for-length, weight-for-height and body mass index-for-age. Methods and development. Geneva: WHO; 2006.

21. Onis M, Onyango AW, Borghi E, Siyam A, Nishida C, Siekmann J. Development of a WHO growth reference for school-aged children and adolescents. Bulletin of the World Health Organization 2007; 85(9):660-667.

22. DeMayer E, Dallman P, Gurney JM, Hallberg L, Sood SK, Srikantia SG. Preventing and controlling iron deficiency anaemia through primary health care: a guide for health administrators and programme managers. Geneva: World Health Organization (WHO); 1989.

23. Ribeiro MCSA, Barata RB, Almeida MF, Silva ZP. Perfil sociodemográfico e padrão de utilização de serviços de saúde para usuários e não-usuários do SUS - PNAD 2003. Cien Saude Colet 2006; 11(4):10111022.

24. Instituto Brasileiro de Geografia e Estatística (IBGE). Diretoria de Pesquisas. Coordenação de Trabalho e Rendimento. Pesquisa de Orçamentos Familiares 2008-2009: Antropometria e Estado Nutricional de Crianças, Adolescentes e Adultos no Brasil. Rio de Janeiro: IBGE; 2010.
25. Osório MM. Fatores determinantes de anemia em crianças. J Pediatr 2002; 78(4):269-278.

26. Brasil. Ministério da Saúde (MS). Centro Brasileiro de Análise e Planejamento. Pesquisa Nacional de Demografia e Saúde da Criança e da Mulher: PNDS 2006. Dimensões do processo reprodutivo e da saúde da criança. Série G. Estatística e Informação em Saúde. Brasília: MS; 2009.

27. Orlonski S, Dellagrana RA, Rech CR, Araújo EDS. Estado nutricional e fatores associados ao déficit de estatura em crianças atendidas por uma unidade de ensino básico em tempo integral. Rev Bras Cresc Desenvolvimento Hum 2009; 19(1):54-62.

28. McLellan F. Obesity rising to alarming levels around the world. Lancet 2002; 359(9315):1412-1415.

29. Suglia SF, Duarte CS, Chambers EC, Boynton-Jarrett R. Cumulative social risk and obesity in early childhood. Pediatrics 2012; 129(5):e1173-1179.

30. Barry M, Popkin BM. The nutrition transition: an overview of world patterns of change. Nutr Rev 2004; 62(7):S140-143.

31. Vieira RCS, Ferreira HS, Costa ACS, Moura FA, Florêncio TMMT, Torres ZMC. Prevalência e fatores de risco de para anemia em crianças préescolares do Estado de Alagoas, Brasil. Rev Bras Saude Matern Infant 2010; 10(1):107-116.

32. Bueno MB, Selem SSC, Arêas JAG, Fisberg RM. Prevalência e fatores associado à anemia entre crianças atendidas em creches públicas de São Paulo. Rev Bras Epidemiol 2006; 9(4):462-470.

33. Monteiro CA, Szarfarc SC, Mondini L. Tendência secular da anemia na infância na Cidade de São Paulo (1984-1996). Rev Saude Publica 2000; 34(6):6272.

34. Filho MB, Souza AI, Miglioli TC, Santos MC. Anemia e obesidade: um paradoxo da transição nutricional brasileira. Cad Saude Publica 2008; 24(2):247-57.

35. Ganz T, Nemeth E. Iron metabolism: interactions with normal and disordered erythropoiesis. Cold Spring Harb Perspect Med 2012; 2(5):a011668.

36. Nemeth E, Rivera S, Gabayan V, Keller C, Taudorf $\mathrm{S}$, Pedersen BK, et al. IL-6 mediates hypoferremia of inflammation by inducing the synthesis of the iron regulatory hormone hepcidin. J Clin Invest 2004; 114(9):1271-1276.

37. Frazer DM, Anderson GJ. The orchestration of body iron intake: how and where do enterocytes receive their cues? Blood, Cells, Molecules, and Disease 2003; 30(3):288-297.

38. Rocha DS, Lamounier JA, Franceschini SCC, Capanema FD, Norton RC, Oliveira VB, Sadala PV, Campos SF, Toni FA, Oliveira LS. Fatores de risco para déficit nutricional em crianças matriculadas em creches. Rev Pediatr 2007; 8(1):17-26.

Artigo apresentado em 25/03/2012

Aprovado em 24/05/2012

Versão final apresentada em 05/06/2012 